\title{
Comparaison entre des prismes de différents facteurs de surface terrière pour établir la structure des diamètres d'un peuplement inéquienne
}

\author{
par Yvon Grenier ${ }^{1}$ et Marc Du Sablon
}

\begin{abstract}
Nous avons fait un inventaire intégral dans une érablière inéquienne de 18 ha pour en déterminer la structure des diamètres. Nous avons aussi pris 72 points d'échantillonnage avec des prismes de facteur de surface terrière de $2,3,4$ et $5 \mathrm{~m}^{2} /$ ha. En simulant un échantillonnage où nous faisons varier le nombre et la dispersion des points de prisme, il appert que c'est le prisme de facteur 2 qui donne les résultats les plus précis et qu'il faut faire deux points d'échantillonnage par hectare pour qu'un échantillon quelconque ait $90 \%$ de probabilité de représenter ce peuplement, sous un seuil de confiance $\alpha=0,05$. De plus, les unités d'échantillonnage doivent être dispersées le plus possible sur le terrain.
\end{abstract}

A complete forest survey was conducted on an 18-ha unevenaged maple stand to derive its diameter structure. Seventy-two sampling points were also established in the same area using prisms of basal area factor of $2,3,4$ and $5 \mathrm{~m}^{2} / \mathrm{ha}$. Simulation of sampling was performed by varying number and dispersion of points. Two sampling points per hectare with a prism of basal area factor of $2 \mathrm{~m}^{2} /$ ha gave the best results in representing this entire stand at a probability level of $90 \%$, with a confidence level of $\alpha=0.05$. Sampling units have to be scattered as much as possible in the forest area to be surveyed.

\section{Introduction}

La méthode d'inventaire par points d'échantillonnage, proposée par Bitterlich (1984) en 1947, permet d'évaluer promptement la surface terrière d'un peuplement. Cependant, on lui prête aussi d'autres usages comme la détermination du nombre de tiges par classe de diamètre (Bonnor et Magnussen 1987).

L'acquisition de ces données trouve immédiatement son application pour les coupes de jardinage. Pour les réaliser, il faut prélever le bon nombre de tiges par classe de diamètre dans le but de conserver au peuplement traité une structure inéquienne équilibrée (Majcen et al. 1990). La méthode d'inventaire par points d'échantillonnage peut donc être utilisée pour ces travaux afin de déterminer la structure de la forêt à aménager.

À ce sujet, Grenier et al. (1991) ont réalisé une étude dans une érablière inéquienne où ils ont conclu que deux points de prisme par hectare permettraient de retrouver la structure de ce peuplement. Ce dernier avait une surface de 18 ha et l'inventaire avait été réalisé avec un prisme de facteur 2 .

L'utilisation de prismes à bas facteur de surface terrière implique que plusieurs arbres seront retenus dans un point d'échantillonnage et que, par conséquent, plusieurs d'entre eux seront jugés douteux par l'opérateur. Un arbre est considéré comme douteux si son image dans le prisme ne permet pas de l'accepter ou de le rejeter avec une assurance absolue. Dans ce cas, il faut procéder à des mesures rigoureuses du diamètre à hauteur de poitrine (dhp) de la tige et de la distance entre celle-ci et le point d'échantillonnage. Ensuite, pour vérifier si cet arbre doit être rejeté ou retenu, on utilise la formule de Bitterlich (1984) $f \times D(\mathrm{~cm}) \geq R(\mathrm{~m})$, où $f$ est une constante liée au facteur de surface terrière du prisme utilisé, $D$, le dhp précis de l'arbre douteux vu du point d'échantillonnage, et $R$, la distance entre le point d'échantillonnage et le centre de cet arbre.

Gouvernement du Québec, Ministère des Forêts, Direction de la recherche, 2700 rue Einstein, Sainte-Foy (Québec), Canada G1P 3W8.

${ }^{1}$ Adresse actuelle: Parcs Canada, C.P. 1180, Havre-Saint-Pierre, Québec, Canada GOG 1P0.
Cette opération est essentielle pour établir la structure des diamètres du peuplement à aménager, car il y a peu de chances que l'acceptation d'un arbre qui aurait dû être rejeté soit compensée par le rejet d'un arbre qui aurait dû être accepté (Grenier et al. 1991). Pour alléger le travail, on peut utiliser des prismes dont les facteurs de surface terrière sont élevés: ainsi, il y aura moins d'arbres douteux (Rondeux 1983). Par contre, la fiabilité des résultats risque d'en souffrir.

Le but de la présente étude est de comparer l'efficacité de prismes de facteur de surface terrière de 2,3 , 4 et $5 \mathrm{~m}^{2} /$ ha pour déterminer lequel permet de reconstituer le plus fidèlement la structure des diamètres du peuplement à aménager.

\section{Matériel et méthodes}

La description détaillée du protocole expérimental a déjà été publiée (Grenier et al. 1991). Cependant, pour réaliser le présent travail, nous avons relocalisé exactement les 72 points d'échantillonnage qui avaient été distribués systématiquement dans une érablière à bouleau jaune et hêtre à grandes feuilles (Betulo luteae-Aceretum sacchari Grandtner 1962 fagetosum grandifoliae Lemieux 1964) de 18 ha à Duchesnay (Canada: $46^{\circ} 55^{\prime} 40^{\prime \prime} \mathrm{N}$. et $71^{\circ} 37^{\prime} 30^{\prime \prime} 0$ ). Dans cette érablière, nous avons fait un inventaire intégral de toutes les tiges marchandes.

À chacun des points d'échantillonnage, nous avons utilisé des prismes de facteur de surface terrière de $2,3,4$ et $5 \mathrm{~m}^{2} /$ ha pour évaluer si un arbre était rejeté ou retenu dans un point d'échantillonnage. Pour tout arbre retenu, nous avons mesuré son dhp et avons compilé les résultats par classe de $10 \mathrm{~cm}$, toutes essences confondues. Les données d'inventaire sont converties en nombre de tiges par hectare par classe de diamètre à l'aide des facteurs d'arbre publiés par Grenier et Lapointe (1991), ce qui permet d'obtenir la structure des diamètres des échantillons.

Nous désirons vérifier si un échantillon quelconque tiré de la population connue a une structure semblable à celle de cette même population, et ce pour les quatre facteurs de 


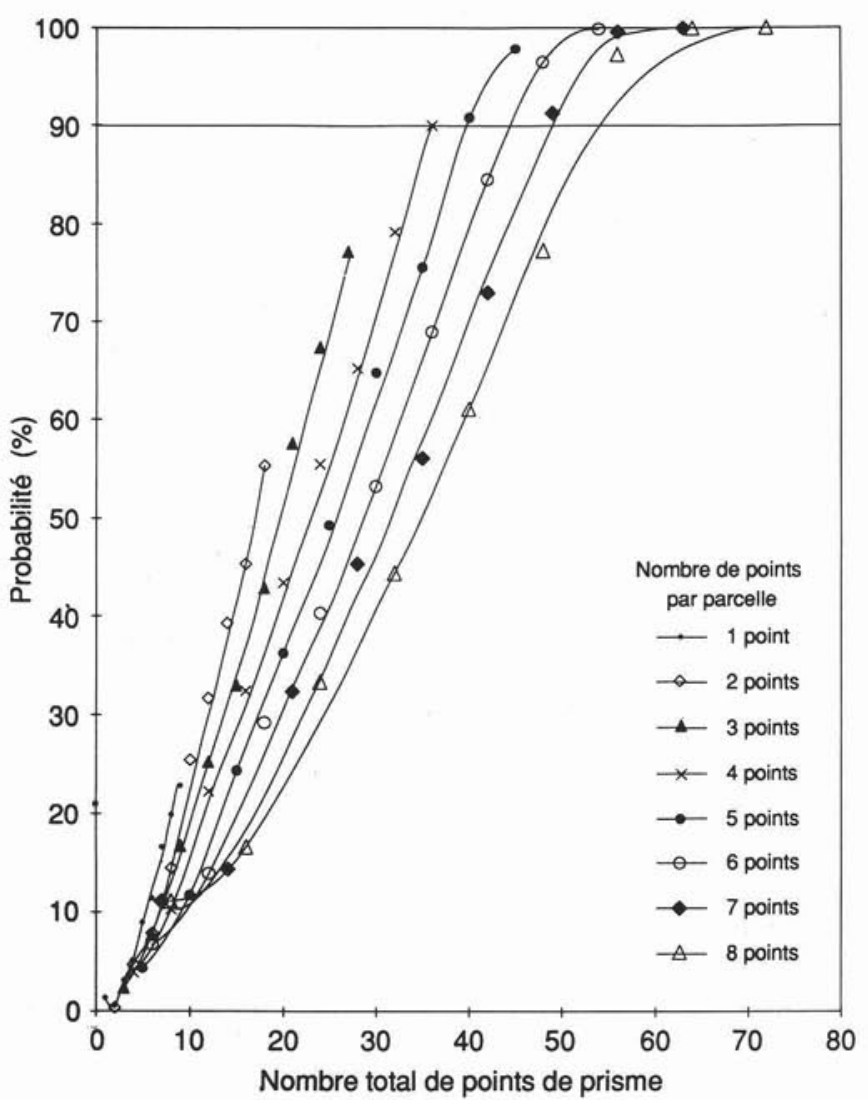

Figure 1. Probabilité qu'un échantillon représente la population au seuil $\alpha=0,05$ pour des nombres de points de prisme provenant de combinaisons aléatoires pris avec un prisme de facteur 2 .

surface terrière. Pour vérifier cette hypothèse, nous utilisons un test d'ajustement, $\chi^{2}$ (Steel et Torrie 1980), et compilons le pourcentage des cas où l'hypothèse n'est pas rejetée, sous un seuil $\alpha=0,05$, pour des échantillons confectionnés de différents nombres de points de prisme. Le paramètre à vérifier est le nombre de tiges par classe de diamètre.

Les échantillons sont composés de $p$ points de prisme par parcelle (de 2 ha chacune), eux-mêmes tirés de $P$ parcelles (constituant tout le peuplement). Le nombre d'échantillons $(N)$ qu'il est possible de construire, selon le nombre de points de prisme et le nombre de parcelles qu'on désire prendre à la fois est le résultat de

$$
N=\left[C\left(\begin{array}{l}
9 \\
P
\end{array}\right)\right] \times\left[C\left(\begin{array}{l}
8 \\
p
\end{array}\right)\right]^{p}
$$

$C$ ( ) représente le nombre de combinaisons possibles pour les variables à l'intérieur des parenthèses, $P$ est le nombre de parcelles $(1 \leq P \leq 9)$ et $p$ est le nombre de points de prisme par parcelle $(1 \leq p \leq 8)$.

Devant la multitude de combinaisons possibles, nous avons dû procéder par échantillonnage selon un procédé de semihasard. La première étape de ce procédé sélectionne toutes les combinaisons possibles de parcelles. Ensuite, pour chaque combinaison de parcelles, les points de prisme sont tirés au hasard, sans remise. Le nombre de tirages par combinaison de parcelle est calculé de façon à ce que nous puissions tester 1008 échantillons par groupe de $P$ parcelles avec chacune $p$ points de prisme, sauf pour les cas où $p=8$ où nous avons

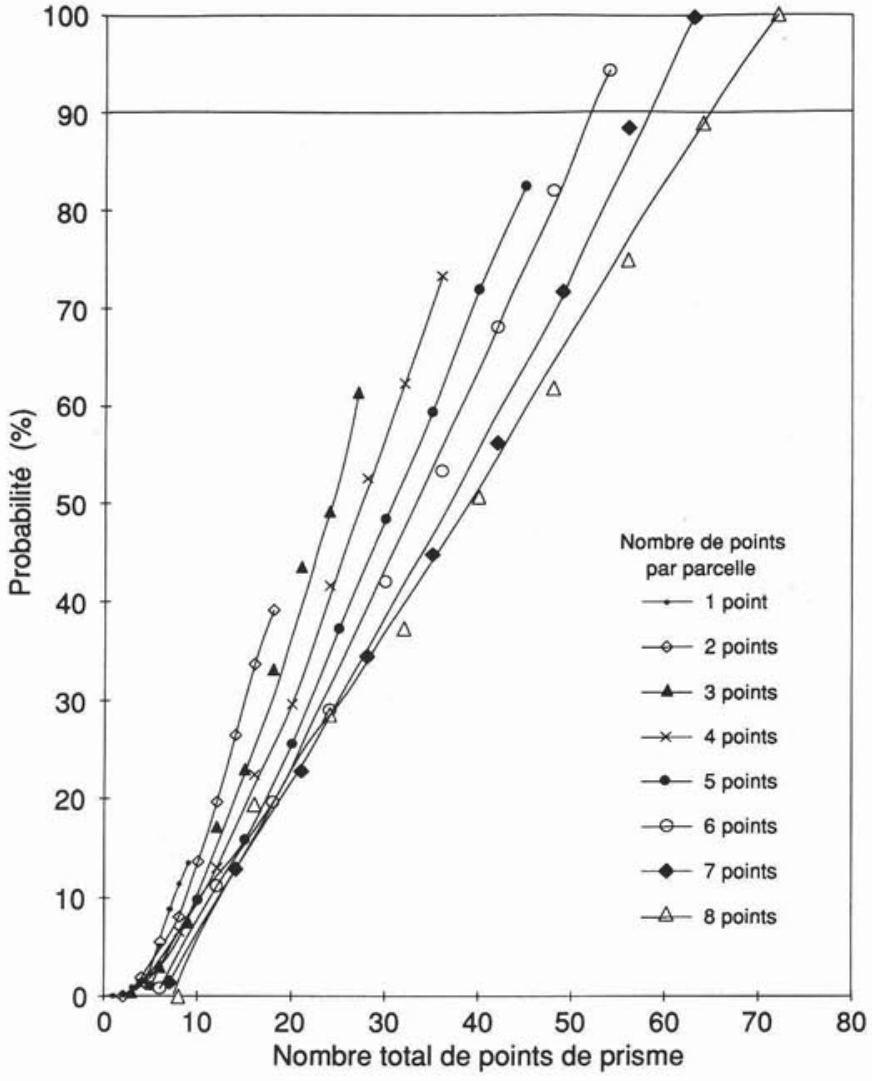

Figure 2. Probabilité qu'un échantillon représente la population au seuil $\alpha=0,05$ pour des nombres de points de prisme provenant de combinaisons aléatoires pris avec un prisme de facteur 3 .

testé $\left(\begin{array}{l}9 \\ P\end{array}\right)$ échantillons et pour les cas où $P=1$ où nous avons testé $9 \times\left(\begin{array}{l}8 \\ p\end{array}\right)$ échantillons.

\section{Résultats}

Les résultats pour les différents facteurs de prisme sont présentés sur les figures 1 à 5 . Sur l'ordonnée, on trouve la probabilité, en pourcentage, qu'un échantillon quelconque représente la population dont il est issu sous un seuil $\alpha=0,05$ et, sur l'abscisse, le nombre total de points de prisme testés par combinaison, impliquant un à huit points de prisme par parcelle.

Nonobstant le prisme utilisé, il est évident que les meilleurs résultats sont obtenus quand les unités d'échantillonnage sont dispersées au maximum sur le terrain. Tout en gardant constant le nombre total de points de prisme, la probabilité qu'un échantillon représente la population augmente avec le nombre de parcelles choisies pour composer cet échantillon, alors que le nombre de points par parcelle diminue. Pour l'illustrer, prenons la figure 1 où nous étudions le prisme de facteur 2 et prenons un nombre total de 24 points de prisme. Premièrement, les meilleurs résultats sont obtenus avec trois points de prime par parcelles et huit parcelles (67\%), ensuite avec quatre points par parcelle et six parcelles $(56 \%)$, puis avec six points par parcelle et quatre parcelles (40\%) et finalement avec huit points de prisme par parcelle et trois parcelles à la fois $(33 \%)$. 


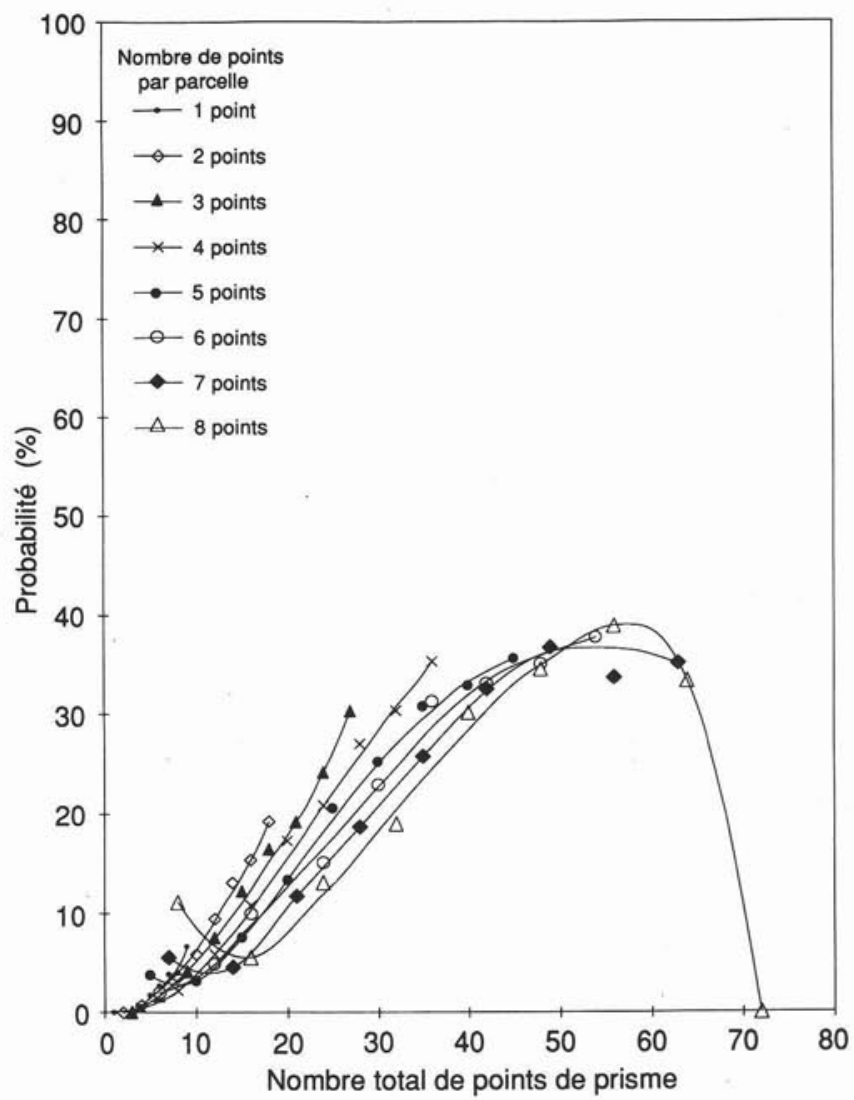

Figure 3. Probabilité qu'un échantillon représente la population au seuil $\alpha=0,05$ pour des nombres de points de prisme provenant de combinaisons aléatoires pris avec un prisme de facteur 4 .

Dans trois de ces quatre figures, nous avons tracé arbitrairement une ligne horizontale à la hauteur de $90 \%$ de probabilité. Pour le prisme de facteur 2 (Figure 1) et pour celui de facteur 3 (Figure 2), les combinaisons impliquant quatre à huit et six à huit points de prisme par parcelle respectivement atteignent ou dépassent ce seuil arbitraire. Pour le prisme de facteur 4 (Figure 3), aucune courbe n'atteint le seuil de $90 \%$ tandis que pour le prisme de facteur 5 (Figure 4), il n'y a que la courbe impliquant huit points de prisme par parcelle qui dépasse le seuil.

La figure 5 reprend, pour chaque facteur de surface terrière, la première courbe par facteur qui atteint ou dépasse le seuil de $90 \%$ de probabilité. Nous avons ajouté aux points expérimentaux les écarts-types par rapport à la moyenne, calculés sur les différentes combinaisons de parcelles étudiées. On remarque que les écarts-types les plus petits sont associés au prisme de facteur 2 tandis que les plus grands sont associés à celui de facteur 5 .

Comme résultat, nous présentons aussi le tableau 1 qui donne un aperçu du nombre d'arbres par classe de diamètre et par facteur de surface terrière, et leur proportion respective, qui ont été retenus lors de l'échantillonnage. C'est le prisme de facteur 2 qui a retenu le plus d'arbres, avec une moyenne de 13,028 $\pm 2,648$ arbres par point. Le coefficient de variation, ramené en pourcentage, donne $20 \%$ pour ce prisme. Il est le plus bas des quatre prismes testés. Le prisme de facteur 3 a retenu une moyenne de $8,778 \pm 2,209$ arbres, soit un coefficient de variation de $25 \%$. Les prismes de

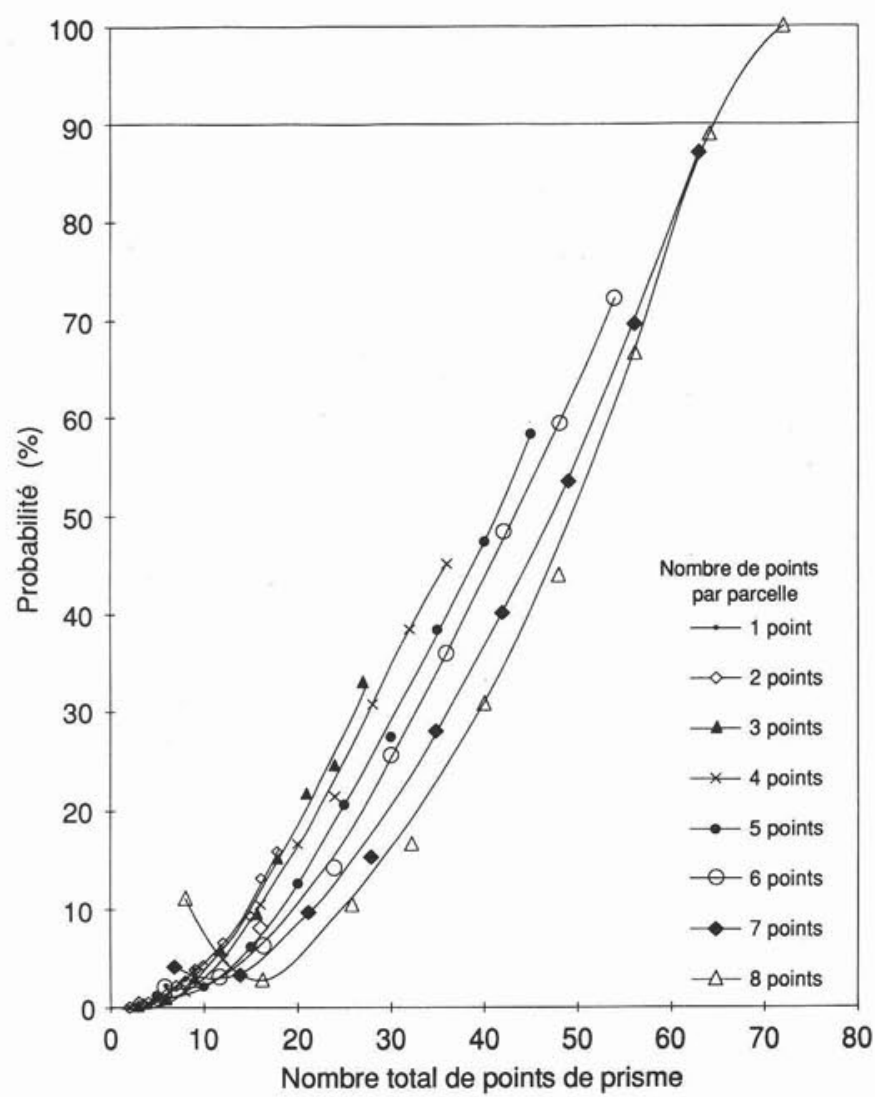

Figure 4. Probabilité qu'un échantillon représente la population au seuil $\alpha=0,05$ pour des nombres de points de prisme provenant de combinaisons aléatoires pris avec un prisme de facteur 5 .

facteur 4 et 5 ont donné respectivement des moyennes de $6,309 \pm 2,209$ et $5,444 \pm 1,845$ arbre par point. Les coefficients de variation associés à ces prismes sont de 32 et $34 \%$ respectivement, ce qui en fait les valeurs les plus élevées de l'étude.

\section{Discussion}

Si on accepte comme satisfaisant une probabilité de $90 \%$ qu'un échantillon quelconque représente la population dont il est issu, on devrait prendre 2, 2,9 et 3,7 points de prisme par hectare, dans ce peuplement de $18 \mathrm{ha}$, avec des prismes de facteur de surface terrière de 2,3 et $5 \mathrm{~m}^{2} /$ ha respectivement. Ces points devraient être dispersés le plus uniformément possible sur le terrain.

On peut se demander lequel de ces trois facteurs de surface terrière serait le plus efficace pour réaliser un tel travail. Certaines personnes ont des contraintes de temps ou d'argent, alors que d'autres ont besoin du maximum de précision. Ce qui demande le plus de temps dans un inventaire au prisme, c'est la vérification des arbres douteux. Leur nombre est, à priori, inconnu. Cependant, on peut supposer que la proportion d'arbres douteux est la même, quel que soit le prisme utilisé. Supposons, pour cet exercice, que tous les arbres soient douteux; dans ce cas, il faudrait vérifier 469 arbres pour le prisme de facteur 2, 456 arbres pour celui de facteur 3 et 359 arbres pour celui de facteur 5 .

Il y a donc peu de différences entre les prismes de facteurs 2 et 3 , sauf pour la précision. Rappelons que le coefficient 


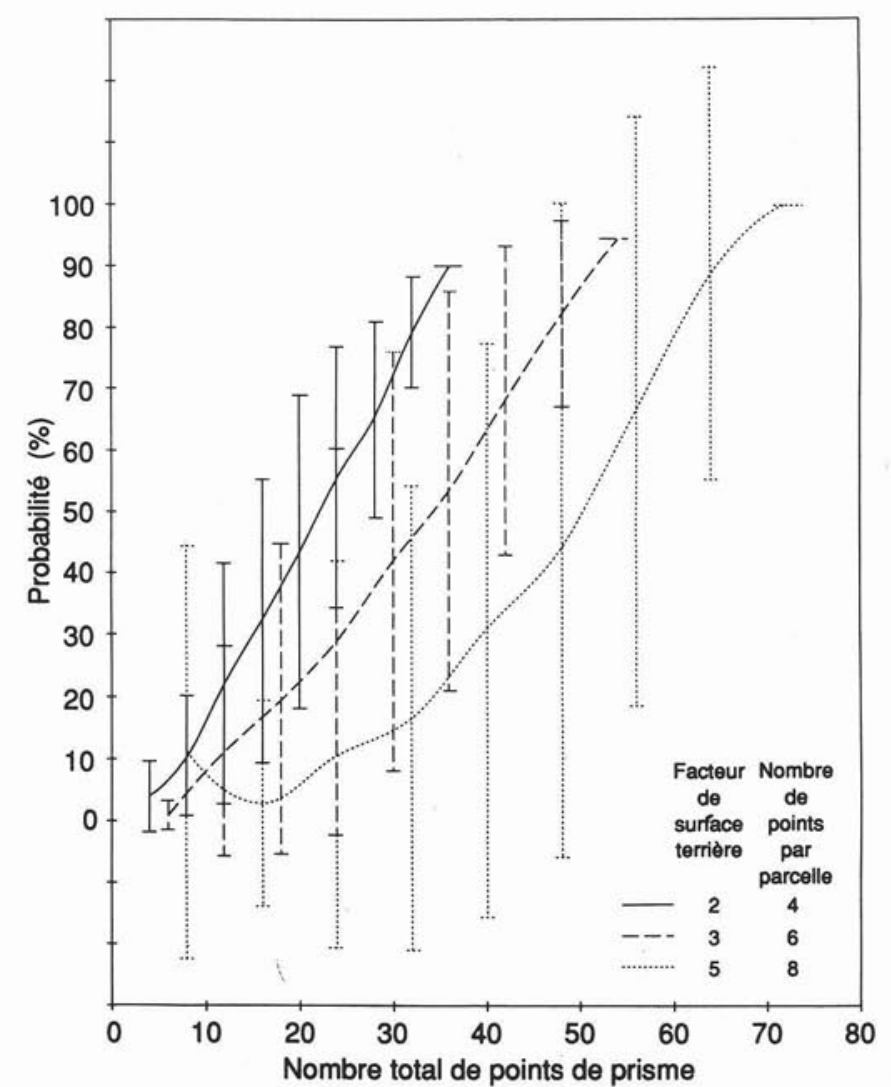

Figure 5. Probabilité qu'un échantillon représente la population au seuil $\alpha=0,05$ pour des nombres de points de prisme provenant de combinaisons aléatoires pris avec des prismes de facteur 2, 3 ou 5. Les barres verticales correspondent à \pm 1 écart-type.

de variation du nombre d'arbres par point d'échantillonnage est plus élevé avec le prisme de facteur 3 qu'avec celui de facteur 2. Nous suggérerions donc d'opter pour le prisme de facteur de surface terrière de $2 \mathrm{~m}^{2} / \mathrm{ha}$.

L'utilisation d'un prisme de facteur 5 permettrait d'effectuer le travail le plus rapidement avec plus de 100 arbres en moins à vérifier. Cependant, une erreur relative importante est associée à cet outil, comme le démontre la figure 5 par les écarts-types. Malgré la rapidité accrue d'exécution, nous ne croyons pas qu'il vaille la peine d'y sacrifier la précision.

En ce sens, la figure 3 (facteur de surface terrière de $4 \mathrm{~m}^{2} / \mathrm{ha}$ ) vient appuyer l'affirmation précédente. La forme des courbes impliquant un ou deux points de prisme par parcelle suit l'allure générale des courbes présentées dans cette étude. Ainsi, les meilleures probabilités sont associées au prisme de facteur 2 et ces résultats décroissent régulièrement jusqu'au prisme de facteur 5. Cependant, dès qu'on travaille avec trois points et plus par parcelle, la régularité disparaît. Le prisme de facteur 5 donne souvent de meilleures probabilités que celui de facteur 4 . Ce phénomène s'accentue en augmentant le nombre de points de prisme par parcelle. Un fait remarquable est une probabilité de $0 \%$ associée à 72 points de prisme pour le prisme de facteur 4 , alors que les trois autres ont donné un probabilité de $100 \%$. À ce sujet, il faut noter qu'il n'y a qu'une seule combinaison possible pour constituer cet échantillon. Nous croyons que le hasard a été favorable dans le cas du prisme de facteur 5 et défavorable dans celui du prisme de facteur 4 . De plus, les très forts écarts-types associés aux prismes de facteurs 4 et 5 militent en leur défaveur. Un appui supplémentaire en ce sens peut être fourni par l'erreur relative sur la surface terrière qu'on peut estimer avec ces outils. La surface terrière réelle de ce peuplement est $25,01 \mathrm{~m}^{2} /$ ha (Grenier et al. 1991). Pour le prisme de facteur 4 , la surface terrière estimée est $27,61 \mathrm{~m}^{2} /$ ha, ce qui amène une erreur relative de $10,4 \% \mathrm{si}$ on prend 72 points de prisme. Pour le prisme de facteur 5 et les mêmes conditions, la surface terrière estimée est $27,22 \mathrm{~m}^{2} / \mathrm{ha}$; l'erreur relative est donc de $8,8 \%$. Les prismes de facteurs 2 et 3 donnent respectivement 26,06 et $26,33 \mathrm{~m}^{2} / \mathrm{ha}$, soit des erreurs relatives de 4,2 et $6,5 \%$.

\section{Conclusion}

L'ensemble des observations décrites dans cet article nous portent à choisir le prisme de facteur de surface terrière de $2 \mathrm{~m}^{2} /$ ha comme étant le meilleur outil parmi les quatre utilisés pour établir la structure des diamètres de cette érablière inéquienne de 18 ha. Pour réaliser un tel inventaire, il faut prendre deux points de prisme par hectare et les points d'échantillonnage doivent être dispersés le plus possible sur le terrain.

\begin{tabular}{|c|c|c|c|c|c|c|c|c|}
\hline \multirow{3}{*}{$\begin{array}{l}\text { Classe de } \\
\text { diamètre } \\
(\mathrm{cm})\end{array}$} & \multicolumn{8}{|c|}{ Facteur de surface terrière $\left(\mathrm{m}^{2} / \mathrm{ha}\right)$} \\
\hline & \multicolumn{2}{|c|}{2} & \multicolumn{2}{|c|}{3} & \multicolumn{2}{|c|}{4} & \multicolumn{2}{|c|}{5} \\
\hline & Nombre & $\%$ & Nombre & $\%$ & Nombre & $\%$ & Nombre & $\%$ \\
\hline 15 & 132 & 14,07 & 92 & 14,56 & 76 & 15,29 & 56 & 14,29 \\
\hline 25 & 211 & 22,49 & 140 & 22,15 & 107 & 21,53 & 83 & 21,17 \\
\hline 35 & 292 & 31,13 & 189 & 29,91 & 146 & 29,38 & 119 & 30,36 \\
\hline 45 & 181 & 19,30 & 119 & 18,83 & 92 & 18,51 & 72 & 18,37 \\
\hline 55 & 75 & 8,00 & 56 & 8,86 & 46 & 9,26 & 35 & 8,93 \\
\hline 65 & 29 & 3,09 & 20 & 3,16 & 18 & 3,62 & 16 & 4,08 \\
\hline 75 & 18 & 1,92 & 16 & 2,53 & 12 & 2,41 & 11 & 2,81 \\
\hline Total & 938 & 100 & 632 & 100 & 497 & 100 & 392 & 100 \\
\hline \multicolumn{9}{|l|}{ Moyenne par } \\
\hline (Écart-type) & \multirow{2}{*}{\multicolumn{2}{|c|}{2,648}} & \multirow{2}{*}{\multicolumn{2}{|c|}{2,209}} & \multirow{2}{*}{\multicolumn{2}{|c|}{2,209}} & \multirow{2}{*}{\multicolumn{2}{|c|}{1,845}} \\
\hline (Coefficient & & & & & & & & \\
\hline de variation) & \multicolumn{2}{|c|}{20} & \multicolumn{2}{|c|}{25} & \multicolumn{2}{|c|}{32} & \multicolumn{2}{|c|}{34} \\
\hline
\end{tabular}




\section{Remerciements}

Nous tenons à remercier Jean-Marc Boivin qui nous a assisté lors de la prise des données; Louis Blais qui nous a conseillé sur l'interprétation statistique des résultats; Fabien Caron, Yvon Richard et Jean Bégin (Université Laval) qui ont revisé et critiqué le manuscrit; Michelle Therrien et Sylvie Bourassa qui ont dactylographié le texte. Les fonds pour la réalisation de ce travail ont été fournis par le ministère des Forêts du Québec.

\section{Références}

Bitterlich, W. 1984. The relascope idea. Relative measurements in forestry. Commonwealth Agricultural Bureaux. Slough, Angleterre.

Bonnor, G.M. et S. Magnussen. 1987. Forest inventories in Canada: A framework for change. For. Chron. 63: 193-198. Grenier, Y., L. Blais et É. Lavoie. 1991. Aire minimum d'échantillonnage ou nombre de points de prisme nécessaires pour établir la structure d'un peuplement inéquienne. Can. J. For. Res. 21: 1632-1638.

Grenier, Y. et F. Lapointe. 1991. Précisions sur les facteurs d'arbre utilisés lors d'inventaires par points d'échantillonnage des peuplements inéquiennes. For. Chron. 67: 389-392.

Majcen, Z., Y. Richard, M. Ménard et Y. Grenier. 1990. Choix des tiges à marquer pour le jardinage d'érablières inéquiennes. Guide technique. Gouvernement du Québec, Ministère de l'Énergie et des Ressources, Direction de la recherche et du développement. Mémoire $\mathrm{n}^{\circ} 96$.

Rondeux, J. 1983. La méthode de l'angle critique: une conception particulière de l'échantillonnage appliqué aux inventaires forestiers. Ann. Gembloux. 89: 183-200.

Steel, R.G.D. et J.H. Torrie. 1980. Principles and procedures of statistics. A biometrical approach. $2^{\mathrm{e}}$ éd., McGraw-Hill Book Company, New York.

\section{Change of Address}

Date Section

Surname first - no titles

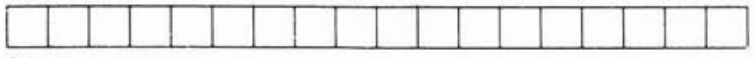

Street

City and Province

Postal Code

\section{Changement d'adresse}

Date Section

Prénom en avant - pas de titres

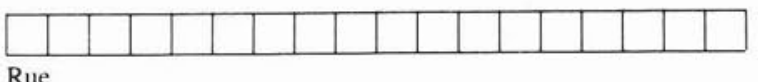

Rue Ville et Province

Code Postale 